ISSN 0103-9954

\title{
FLORÍSTICA E ESTRUTURA DO COMPONENTE ARBÓREO E RELAÇÃO COM VARIÁVEIS AMBIENTAIS EM UM REMANESCENTE FLORESTAL EM CAMPOS NOVOS - SC
}

\author{
FLORISTIC AND STRUCTURE OF THE TREE COMPONENT AND RELATION WITH \\ ENVIRONMENTAL VARIABLES IN A FOREST REMNANT IN CAMPOS NOVOS - SC
}

\begin{abstract}
Pedro Higuchi ${ }^{1}$ Ana Carolina da Silva ${ }^{2}$ Tiago de Souza Ferreira ${ }^{3}$ Sheila Trierveiler de Souza ${ }^{4}$ Juliano
Pereira Gomes $^{5}$ Karina Montibeller da Silva ${ }^{6}$ Kristiana Fiorentin dos Santos ${ }^{6}$

Eduardo Jocimar Berndt ${ }^{8}$ Janir de Oliveira Souza Junior ${ }^{6}$ Deise Tatiani de Gois ${ }^{7}$

Felipe Weiduschat ${ }^{7}$
\end{abstract}

\section{RESUMO}

O presente estudo objetivou conhecer os padrões da composição florística e estrutural do componente arbóreo de um trecho de remanescente de Floresta Ombrófila Mista Montana em Campos Novos - SC e determinar as variáveis ambientais que influenciam estes padrões. Para isso, foi amostrado 1 ha de floresta por meio de 50 parcelas de $10 \times 20 \mathrm{~m}$ dispostas de forma sistemática, distanciada $30 \mathrm{~m}$ entre si, no remanescente florestal. Dentro das parcelas foram identificados e mensurados (circunferência medida a altura do peito, CAP, e altura total) $\mathrm{CAP}$, e altura total) todos os indivíduos arbóreos vivos com CAP $\geq 15,7 \mathrm{~cm}$. Os dados ambientais relacionados às propriedades químicas e físicas dos solos e à topografia também foram coletados em cada parcela. Foram calculados o índice de Shannon-Wiener (H'), a equabilidade de Pielou (J') e os estimadores fitossociológicos. A organização florístico-estrutural do fragmento foi analisada por meio de uma NMDS (Nonmetric multidimensional scalling). As variáveis ambientais foram ajustadas a posteriori à ordenação produzida, sendo aquelas significativas $(\mathrm{p}<0,05)$ plotadas na forma de vetores. Foram amostrados 1.027 indivíduos, que totalizaram uma área basal de $43,57 \mathrm{~m}^{2}$, distribuídos em 88 espécies e 41 famílias botânicas. A diversidade do remanescente estudado foi relativamente alta $\left(H^{\prime}=3,59\right)$ e a dominância baixa $\left(\mathrm{J}^{\prime}=0,80\right)$. A espécie de maior VI foi Araucaria angustifolia (Bertol.) Kuntze (14,44\%). A análise multivariada NMDS indicou um gradiente florístico-estrutural relacionado à cota média (altitude), saturação de bases, $\mathrm{pH}$ e teores de $\mathrm{P}$ nos solos.

Palavras-chave: Floresta com araucária; NMDS; variáveis ambientais.

1 Engenheiro Florestal, Dr., Professor Associado do Departamento de Engenharia Florestal, Centro de Ciências Agroveterinárias, Universidade do Estado de Santa Catarina, Av. Luis de Camões, 2090, CEP 88520-000, Lages (SC), Brasil. higuchip@gmail.com

2 Engenheira Florestal, Dra ${ }^{\mathrm{a}}$, Professora Adjunto do Departamento de Engenharia Florestal, Centro de Ciências Agroveterinárias, Universidade do Estado de Santa Catarina, Av. Luis de Camões, 2090, CEP 88520-000, Lages (SC), Brasil.carol_sil4@yahoo.com.br

3 Engenheiro Florestal, MSc., Departamento de Engenharia Florestal, Centro de Ciências Agroveterinárias, Universidade do Estado de Santa Catarina, Av. Luis de Camões, 2090, CEP 88520-000, Lages (SC), Brasil. tiagoferreira@florestal.eng.br

4 Engenheira Florestal, Mestranda em Ciência do Solo, Centro de Ciências Agroveterinárias, Universidade do Estado de Santa Catarina, Av. Luis de Camões, 2090, CEP 88520-000, Lages (SC), Brasil. sheila_tsouza@hotmail.com

5 Engenheiro Florestal, Doutorando em Produção Vegetal, Centro de Ciências Agroveterinárias, Universidade do Estado de Santa Catarina, Av. Luis de Camões, 2090, CEP 88520-000, Lages (SC), Brasil. julianopgomes@yahoo.com.br

6 Engenheira Florestal, MSc., Centro de Ciências Agroveterinárias, Universidade do Estado de Santa Catarina, Av. Luis de Camões, 2090, CEP 88520-000, Lages (SC), Brasil. karynaflorestal@yahoo.com.br/ kristianafiorentin@gmail.com/janir100@hotmail.com

7 Engenheiro (a) Florestal, Centro de Ciências Agroveterinárias, Universidade do Estado de Santa Catarina, Av. Luis de Camões, 2090, CEP 88520-000, Lages (SC), Brasil. alemaofloresta@yahoo.com.br/deisitg@hotmail.com/ felypefe@gmail.com

Recebido para publicação em 12/11/2012 e aceito em 4/02/2014

Ci. Fl., v. 26, n. 1, jan.-mar., 2016 


\begin{abstract}
In order to identify the floristic and structure patterns of the tree component and to determine the environmental variables that influence these patterns in a sector of montane Araucaria Forest remnant, 50 plots of $10 \times 20 \mathrm{~m}$ were allocated, using systematic sampling, $30 \mathrm{~m}$ apart from each other, in Campos Novos, SC. Within the plots, all living trees with cbh (circumference at breast height) $\geq 15,7 \mathrm{~cm}$ were identified and measured (cbh and total height). Environmental data related to chemical and physical properties of soils and topography were also collected from each sampled plot. To describe the tree vegetation richness, diversity and structure, the Shannon-Wiener index (H'), the Pielou evenness index (J') and the phytosociological estimates were obtained. The floristic and structural organization of the fragment was analyzed through a NMDS (Nonmetric Multidimensional Scaling). The environmental variables were fitted a posteriori in the produced ordination, being those significant $(\mathrm{p}<0.05)$ plotted as vectors. A total 1.027 individuals were sampled, which represented a basal area of $43.57 \mathrm{~m}^{2}$, distributed in 88 species and 41 families. The diversity of the fragment studied was relatively high $\left(\mathrm{H}^{\prime}=3.59\right)$ with low dominance $\left(\mathrm{J}^{\prime}=0.80\right)$. The specie with higher VI was Araucaria angustifolia (Bertol.) Kuntze (14.44\%). The NMDS indicated a floristic-structural gradient related to the mean elevation (altitude), soil base saturation, soil $\mathrm{pH}$ and $\mathrm{P}$ soil content.
\end{abstract}

Keywords: araucaria forest; NMDS; environments variables.

\section{INTRODUÇÃO}

A Floresta Ombrófila Mista (FOM), também conhecida como Floresta com Araucária pela ocorrência de Araucaria angustifolia (Bertol.) Kuntze no dossel superior, representa um importante componente da paisagem na região Sul do Brasil. De acordo com o IBGE (2012), a FOM pode ser classificada de acordo com a altitude e a proximidade do curso de água nas formações: Aluvial, Submontana ( $<400 \mathrm{~m})$, Montana (400-1000 m) e Alto-Montana (>1000 m). No estado de Santa Catarina, Klein (1978) propôs a subdivisão da FOM em quatro grupos distintos: i) Floresta de Araucária na Bacia Iguaçu-Negro e na parte superior das bacias dos afluentes do rio Uruguai; ii) Florestas de Araucária na bacia Pelotas-Canoas; iii) Floresta de Araucária do extremo oeste e iv) Núcleos de Pinhais da Mata Pluvial Atlântica.

Os locais de menor altitude, com aproximadamente $600 \mathrm{~m}$ acima do nível do mar ou, como no presente estudo, em torno de 700 $\mathrm{m}$ acima do nível do mar, ambientalmente são caracterizados por apresentarem temperaturas médias anuais relativamente maiores e floristicamente caracterizados por um maior contato com a Floresta Estacional Decidual e Semidecidual, resultando numa mistura de elementos destas duas fitofisionomias e, consequentemente, numa elevada riqueza florística para os padrões regionais (HIGUCHI et al., 2012b).
Tendo em vista que essas áreas passaram, nas últimas décadas, por um intenso processo de fragmentação e perturbação, estudos que tenham como proposta subsidiar estratégias de conservação e o manejo sustentável é de grande relevância. Um dos grandes questionamentos se refere ao entendimento de como as espécies arbóreas se distribuem em função de gradientes ambientais. Dentre as principais variáveis ambientais que afetam a estruturação do componente arbóreo em pequenas escalas espaciais, destacam-se as edáficas e topográficas. De acordo com Clark (2002), as variáveis edáficas, representadas principalmente pelo o $\mathrm{pH}$, quantidade de nutriente e textura dos solos, variam de acordo com a topografia e influenciam a distribuição das plantas. Considerando que no estado de Santa Catarina são raros os estudos que investigam a relação entre os padrões estruturais do componente arbóreo da FOM e variáveis ambientais (e.g. PUCHALSKI, 2004; HIGUCHI et al., 2012a; HIGUCHI et al., 2013), os objetivos deste trabalho foram: i) conhecer os padrões da composição florística e estrutural do componente arbóreo de um trecho de remanescente de Floresta Ombrófila Mista Montana em Campos Novos, SC, e ii) determinar as variáveis ambientais que influenciam estes padrões.

\section{MATERIAL E MÉTODOS}

O presente estudo foi realizado em um trecho de remanescente florestal que possui um total 
de 1.551,37 ha, localizado no Município de Campos Novos - SC, na latitude $27^{\circ} 35^{\prime} 26.7432^{\prime}$ 'S, longitude $51^{\circ} 11^{\prime} 5.8518^{\prime \prime} \mathrm{W}$ e altitude aproximada de $700 \mathrm{~m}$. A área faz vizinhança ao Parque Estadual do Rio Canoas e está localizada nas margens do reservatório de água criado pela Usina Hidrelétrica de Campos Novos, ocupando um gradiente topográfico. O local pertence à Bacia Hidrográfica do Rio Uruguai e, de acordo com o banco de dados WordClim (HIJMANS et al., 2005), a precipitação média anual é de $1.719 \mathrm{~mm}$, com chuvas bem distribuídas durante o ano, e a temperatura média anual é de $17,1^{\circ} \mathrm{C}$. A vegetação da região é classificada, segundo o IBGE (2012), como Floresta Ombrófila Mista (FOM). A área é formada por partes em estágios tardios e iniciais de sucessão e representa um dos poucos remanescentes com um trecho de grande extensão de área contínua primária de Floresta Ombrófila Mista presente no estado de Santa Catarina, o que reforça sua importância em termos de conservação da diversidade biológica.

Os dados foram coletados em 50 parcelas de $10 \times 20 \mathrm{~m}\left(200 \mathrm{~m}^{2}\right)$, dispostas de forma sistemática, distanciadas $30 \mathrm{~m}$ entre si (Figura 1). A área total amostrada foi de 1 ha.
Dentro das parcelas foram avaliados todos os indivíduos arbóreos vivos com circunferência na altura do peito (medido a $1,30 \mathrm{~m}$ do solo, CAP) $\geq 15,7 \mathrm{~cm}$. Esses indivíduos foram identificados e tiveram seus CAPs mensurados, com posterior transformação para diâmetro a altura do peito (DAP). Indivíduos com troncos múltiplos foram medidos quando a raiz da soma dos quadrados dos DAPs foi maior do que $5 \mathrm{~cm}$. Foram realizados caminhamentos no remanescente e, espécies não amostradas nas parcelas, foram identificadas e computadas, de forma a complementar a riqueza florística da área. As identificações foram realizadas por meio de comparações em herbários e literatura e as espécies de angiospermas foram delimitadas nas famílias de acordo com o sistema APG III (ANGIOSPERM PHYLOGENY GROUP, 2009).

Os dados ambientais relacionados às propriedades químicas e físicas dos solos e à topografia também foram coletados em cada parcela amostrada. Foi obtida uma amostra composta de solos em cada parcela, oriunda de quatro coletas realizadas no perfil de 0 a $20 \mathrm{~cm}$. Destas, foram obtidas as propriedades químicas e físicas dos solos em análises realizadas no Laboratório de

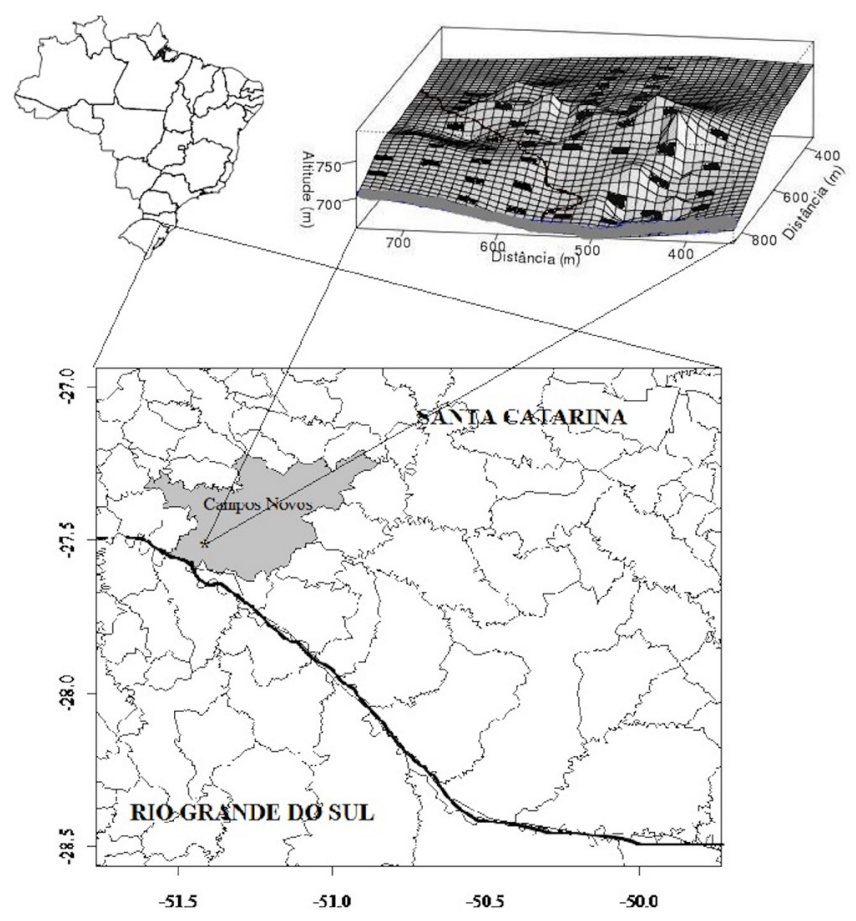

FIGURA 1: Localização da área de estudo e das parcelas alocadas no trecho do remanescente florestal, ao longo do gradiente topográfico, no município de Campos Novos - SC.

FIGURE 1: Location of study area and sample plots in a sector of forest remnant, along the topographic gradient, of the municipality of Campos Novos - SC. 
Solos do Centro de Ciências Agroveterinárias da Universidade do Estado de Santa Catarina-UDESC, de acordo com as recomendações EMBRAPA (1997). Foram quantificados o $\mathrm{pH}$, fósforo, potássio, cálcio, magnésio e alumínio do solo, e calculados os índices: $\mathrm{H}+\mathrm{Al}, \mathrm{t}$ (CTC efetiva), $\mathrm{T}$ (CTC a $\mathrm{pH}=7,0$ ), SB (soma de bases trocáveis) e $\mathrm{V}$ (saturação de bases da CTC a pH = 7,0). Três variáveis topográficas foram coletadas em cada parcela: cota, desnível máximo e declividade média. A cota foi considerada como a média dos valores de altitude de cada um dos vértices de cada parcela. O desnível máximo correspondeu à maior distância vertical entre os vértices das parcelas (OLIVEIRA FILHO et al., 1994). A declividade média foi calculada por meio da média simples das declividades dos quatro lados da parcela retangular.

A suficiência da amostra para determinar a riqueza de espécies inventariadas foi analisada por meio da curva de acumulação de espécies, construída pelo método de aleatorização, com 1.000 permutações. A distribuição dos valores de riqueza estimados para as unidades amostrais, por meio das permutações, foi inserida na curva de acumulação de espécies, utilizando gráficos do tipo boxplot. Este procedimento foi realizado por meio do programa estatístico R (R DEVELOPMENT CORE TEAM, 2011) e a biblioteca Vegan (OKSANEN et al., 2009). A diversidade do remanescente estudado foi avaliada pelo índice de Shannon-Wiener (H') e a dominância pela equabilidade de Pielou (J') (BROWER e ZAR, 1984), calculados pelo programa R (R DEVELOPMENT CORE TEAM, 2011). Para a caracterização estrutural do remanescente, foram calculados os estimadores fitossociológicos para cada espécie (MUELLER-DOMBOIS e ELLEMBERG, 1974): densidade absoluta e relativa, frequência absoluta e relativa, dominância absoluta e relativa e valor de importância (VI).

A organização florística e estrutural do fragmento foi analisada por meio da técnica de análise multivariada NMDS (Nonmetric multidimensional scalling) (MINCHIN, 1987). A adequabilidade da ordenação para a análise foi avaliada por meio do valor de stress. Este é um método de ordenação não paramétrico, que plota as parcelas em um gráfico de dispersão, de forma que as distâncias euclidianas entre as parcelas são proporcionais à dissimilaridade entre elas (BABWETEERA e BROWN, 2009). Os vetores das variáveis significativas $(p<0,05)$ foram plotados a posteriori, utilizando a função envifit (OKSANEN et al., 2009). A análise NMDS foi realizada no programa R (R DEVELOPMENT CORE TEAM, 2011), por meio da biblioteca Vegan (OKSANEN et al., 2009).

\section{RESULTADOS E DISCUSSÃO}

No remanescente estudado foram encontradas 95 espécies (dentro das parcelas e no caminhamento), sendo que cinco destas foram identificadas somente em nível de família e duas em nível de gênero (Tabela 1). As espécies identificadas estão distribuídas em 72 gêneros e 44 famílias botânicas. As famílias com maior número de espécies foram Myrtaceae (10), Fabaceae (8), Lauraceae (8), Salicaceae (6), Euphorbiaceae (4), Meliaceae (4), Primulaceae (4), Rutaceae (4) e Sapindaceae (4). Os gêneros com maior riqueza de espécies foram Myrsine (4), Zanthoxylum (3) e Solanum (3). A família Myrtaceae, com o maior número de espécie, também se destacou como a mais importante em outros trabalhos na FOM ou em áreas de ecótonos com influência da FOM (SEGER et al., 2005; KOZERA et al., 2006; LINGNER et al., 2007; HIGUCHI et al., 2012a; HIGUCHI et al., 2012b; SILVA et al., 2012; CORDEIRO et al., 2013; HIGUCHI et al., 2013; FERREIRA, et al., 2013; SILVA et al., 2013), demonstrando ser uma família de grande riqueza na região. Porém, geralmente não é comum o grande número de espécies de Fabaceae na FOM, como o encontrado no presente estudo. Essa riqueza elevada se deve à influência da Floresta Estacional, já que a família Fabaceae é frequentemente encontrada como a de maior riqueza neste tipo de fitofisionomia na região sul do Brasil (e.g. VACCARO et al., 1999; JARENKOW e WAECHTER, 2001). A influência da Floresta Estacional é confirmada pela presença de espécies comuns nesta fitofisionomia, como Aspidosperma australe Müll.Arg., Helietta apiculata Benth., Myrocarpus frondosus Allemão, Parapiptadenia rigida (Benth.) Brenan, Cordia americana (L.) Gottschling \& J.J.Mill., Schaefferia argentinensis Speg. e Cordyline spectabilis Kunth \& Bouché. Desta forma, o perfil florístico do remanescente estudado é caracterizado pela mistura da flora arbórea oriunda da FOM e da Floresta Estacional, caracterizando uma área de elevada riqueza de espécies arbóreas e de grande importância conservacionista.

Dentro das parcelas foram amostrados 1.027 indivíduos, distribuídos em 88 espécies, 67 gêneros e 41 famílias botânicas (Tabela 2). Dois indivíduos não puderam ser identificados devido 
TABELA 1: Espécies arbóreas encontradas em um remanescente de Floresta Ombrófila Mista Montana, localizado no município de Campos Novos - SC, distribuídas em suas respectivas famílias botânicas.

TABLE 1: Tree species found in a Montane Araucaria Forest remnant, located in Campos Novos - SC, distributed along their respective families.

\begin{tabular}{|c|c|c|}
\hline Famílias & Espécies & Local \\
\hline Adoxaceae & Sambucus australis Cham. \& Schltdl. & $\mathrm{P}$ \\
\hline \multirow{2}{*}{ Anacardiaceae } & Lithraea brasiliensis Marchand & $\mathrm{P}$ \\
\hline & Schinus terebinthifolius Raddi & $\mathrm{F}$ \\
\hline \multirow{2}{*}{ Annonaceae } & Annona rugulosa (Schltdl.) H.Rainer & $\mathrm{P}$ \\
\hline & Annona sylvatica A.St.-Hil. & $\mathrm{P}$ \\
\hline Apocynaceae & Aspidosperma australe Müll.Arg. & $\mathrm{P}$ \\
\hline Aquifoliaceae & Ilex dumosa Reissek & $\mathrm{P}$ \\
\hline Araliaceae & Oreopanax fulvus Marchal & $\mathrm{F}$ \\
\hline Araucariaceae & Araucaria angustifolia (Bertol.) Kuntze & $\mathrm{P}$ \\
\hline Arecaceae & Syagrus romanzoffiana (Cham.) Glassman & $\mathrm{P}$ \\
\hline Asparagaceae & Cordyline spectabilis Kunth \& Bouché & $\mathrm{P}$ \\
\hline \multirow{2}{*}{ Asteraceae } & Piptocarpha angustifolia Dusén & $\mathrm{P}$ \\
\hline & Vernonanthura discolor (Spreng.) H.Rob. & $\mathrm{P}$ \\
\hline Bignoniaceae & Jacaranda puberula Cham. & $\mathrm{P}$ \\
\hline Boraginaceae & Cordia americana (L.) Gottschling \& J.J.Mill. & $\mathrm{P}$ \\
\hline Canellaceae & Cinnamodendron dinisii Schwacke & $\mathrm{P}$ \\
\hline Cannabaceae & Trema micrantha (L.) Blume & $\mathrm{P}$ \\
\hline Cardiopteridaceae & Citronella paniculata (Mart.) R.A.Howard & $\mathrm{P}$ \\
\hline \multirow{2}{*}{ Celastraceae } & Maytenus aquifolia Mart. & $\mathrm{P}$ \\
\hline & Schaefferia argentinensis Speg. & $\mathrm{P}$ \\
\hline Clethraceae & Clethra scabra Pers. & $\mathrm{P}$ \\
\hline Combretaceae & Terminalia australis Cambess. & $\mathrm{P}$ \\
\hline Cunoniaceae & Lamanonia ternata Vell. & $\mathrm{P}$ \\
\hline Cyatheaceae & Cyatheaceae 1 & $\mathrm{P}$ \\
\hline Dicksoniaceae & Dicksonia sellowiana Hook. & $\mathrm{P}$ \\
\hline \multirow[t]{2}{*}{ Elaeocarpaceae } & Sloanea monosperma Vell. & $\mathrm{P}$ \\
\hline & Gymnanthes concolor (Spreng.) Müll.Arg. & $\mathrm{P}$ \\
\hline \multirow{6}{*}{ Euphorbiaceae } & Sapium glandulosum (L.) Morong & $\mathrm{P}$ \\
\hline & Sebastiania brasiliensis Spreng. & $\mathrm{P}$ \\
\hline & Sebastiania commersoniana (Baill.) L. B. Sm. \& Downs & $\mathrm{F}$ \\
\hline & Albizia niopoides (Spruce ex Benth.) Burkart & $\mathrm{P}$ \\
\hline & Dalbergia frutescens (Vell.) Britton & $\mathrm{P}$ \\
\hline & Erythrina falcata Benth. & $\mathrm{P}$ \\
\hline \multirow{5}{*}{ Fabaceae } & Inga sp. & $\mathrm{P}$ \\
\hline & Lonchocarpus campestris Mart. ex Benth. & $\mathrm{P}$ \\
\hline & Mimosa scabrella Benth. & $\mathrm{F}$ \\
\hline & Myrocarpus frondosus Allemão & $\mathrm{P}$ \\
\hline & Parapiptadenia rigida (Benth.) Brenan & $\mathrm{P}$ \\
\hline \multirow[t]{3}{*}{ Lamiaceae } & Vitex megapotamica (Spreng.) Moldenke & $\mathrm{P}$ \\
\hline & Cinnamoтum glaziovii (Mez) Kosterm. & $\mathrm{P}$ \\
\hline & Nectandra megapotamica (Spreng.) Mez & $\mathrm{P}$ \\
\hline \multirow{6}{*}{ Lauraceae } & Ocotea porosa (Mez) L.Barroso & $\mathrm{P}$ \\
\hline & Ocotea pulchella Mart. & $\mathrm{P}$ \\
\hline & Persea willdenowii Kosterm. & $\mathrm{P}$ \\
\hline & Lauraceae 1 & $\mathrm{P}$ \\
\hline & Lauraceae 2 & $\mathrm{P}$ \\
\hline & Lauraceae 3 & $\mathrm{P}$ \\
\hline
\end{tabular}


TABELA 1: Continuação...

TABLE 1: Continued...

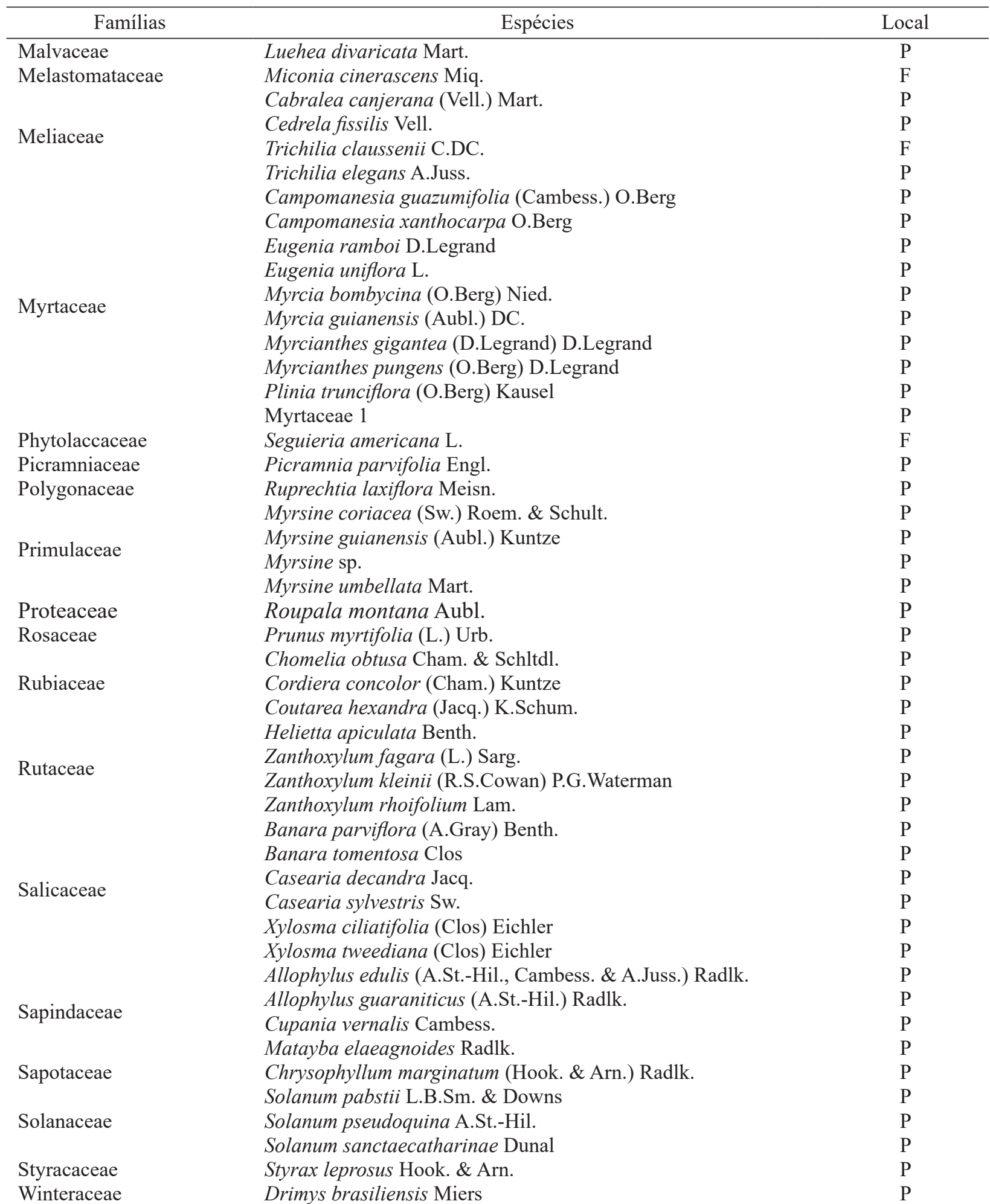

Em que: $\mathrm{P}=$ espécies encontradas dentro das parcelas; $\mathrm{F}$ = espécies não amostradas nas parcelas, encontradas somente nos caminhamentos.

à impossibilidade de coleta de material botânico, em função de estarem sem folhas no período do levantamento. A curva de acumulação de espécies amostradas nas parcelas indicou uma amostragem adequada (Figura 2), sendo que houve um aumento de apenas $0,75 \%$ no número de espécies com a 
TABELA 2: Descritores fitossociológicos de espécies amostradas em um remanescente de Florestal Ombrófila Mista Montana no Município de Campos Novos - SC, ordenadas pelo valor de importância (VI, em \%).

TABLE 2: Phytosociological descriptors of species sampled in a remnant of Montane Araucaria Forest in Municipality of Campos Novos - SC, ranked by importance value (VI, \%).

\begin{tabular}{|c|c|c|c|c|c|c|c|}
\hline Táxons & DA & DR & DoA & DoR & FA & FR & VI \\
\hline Araucaria angustifolia & 40 & 3,89 & 15,4673 & 35,50 & 44 & 3,94 & 14,44 \\
\hline Cupania vernalis & 120 & 11,68 & 3,2478 & 7,45 & 82 & 7,33 & 8,82 \\
\hline Nectandra megapotamica & 90 & 8,76 & 2,6155 & 6,00 & 64 & 5,72 & 6,83 \\
\hline Luehea divaricata & 45 & 4,38 & 5,0842 & 11,67 & 46 & 4,11 & 6,72 \\
\hline Matayba elaeagnoides & 40 & 3,89 & 3,4028 & 7,81 & 40 & 3,58 & 5,09 \\
\hline Allophylus edulis & 64 & 6,23 & 0,6882 & 1,58 & 56 & 5,01 & 4,27 \\
\hline Lauraceae 3 & 33 & 3,21 & 1,6026 & 3,68 & 36 & 3,22 & 3,37 \\
\hline Sebastiania brasiliensis & 59 & 5,74 & 0,3648 & 0,84 & 32 & 2,86 & 3,15 \\
\hline Trichilia elegans & 42 & 4,09 & 0,1314 & 0,30 & 44 & 3,94 & 2,78 \\
\hline Banara tomentosa & 32 & 3,12 & 0,3949 & 0,91 & 42 & 3,76 & 2,59 \\
\hline Cordyline spectabilis & 41 & 3,99 & 0,1894 & 0,43 & 30 & 2,68 & 2,37 \\
\hline Cedrela fissilis & 16 & 1,56 & 1,2929 & 2,97 & 22 & 1,97 & 2,16 \\
\hline Helietta apiculata & 21 & 2,04 & 0,8412 & 1,93 & 28 & 2,50 & 2,16 \\
\hline Myrsine umbellata & 27 & 2,63 & 0,1661 & 0,38 & 32 & 2,86 & 1,96 \\
\hline Parapiptadenia rigida & 25 & 2,43 & 0,3880 & 0,89 & 24 & 2,15 & 1,82 \\
\hline Coutarea hexandra & 22 & 2,14 & 0,3620 & 0,83 & 24 & 2,15 & 1,71 \\
\hline Ocotea pulchella & 8 & 0,78 & 1,2896 & 2,96 & 12 & 1,07 & 1,60 \\
\hline Lonchocarpus campestris & 16 & 1,56 & 0,4243 & 0,97 & 24 & 2,15 & 1,56 \\
\hline Casearia decandra & 15 & 1,46 & 0,1728 & 0,40 & 28 & 2,50 & 1,45 \\
\hline Syagrus romanzoffiana & 14 & 1,36 & 0,4303 & 0,99 & 22 & 1,97 & 1,44 \\
\hline Inga sp. & 17 & 1,66 & 0,1912 & 0,44 & 20 & 1,79 & 1,29 \\
\hline Annona rugulosa & 16 & 1,56 & 0,2216 & 0,51 & 18 & 1,61 & 1,23 \\
\hline Cinnamodendron dinisii & 9 & 0,88 & 0,5374 & 1,23 & 14 & 1,25 & 1,12 \\
\hline Chrysophyllum marginatum & 14 & 1,36 & 0,1656 & 0,38 & 16 & 1,43 & 1,06 \\
\hline Solanum sanctaecatharinae & 12 & 1,17 & 0,0758 & 0,17 & 18 & 1,61 & 0,98 \\
\hline Prunus myrtifolia & 8 & 0,78 & 0,3620 & 0,83 & 12 & 1,07 & 0,89 \\
\hline Dicksonia sellowiana & 9 & 0,88 & 0,2050 & 0,47 & 14 & 1,25 & 0,87 \\
\hline Dalbergia frutescens & 12 & 1,17 & 0,2406 & 0,55 & 8 & 0,72 & 0,81 \\
\hline Casearia sylvestris & 8 & 0,78 & 0,0496 & 0,11 & 16 & 1,43 & 0,77 \\
\hline Myrsine coriacea & 10 & 0,97 & 0,0397 & 0,09 & 14 & 1,25 & 0,77 \\
\hline Myrcianthes pungens & 7 & 0,68 & 0,1589 & 0,36 & 12 & 1,07 & 0,71 \\
\hline Sloanea monosperma & 4 & 0,39 & 0,3829 & 0,88 & 8 & 0,72 & 0,66 \\
\hline Picramnia parvifolia & 7 & 0,68 & 0,0962 & 0,22 & 12 & 1,07 & 0,66 \\
\hline Campomanesia xanthocarpa & 10 & 0,97 & 0,0831 & 0,19 & 8 & 0,72 & 0,63 \\
\hline Schaefferia argentinensis & 8 & 0,78 & 0,0411 & 0,09 & 10 & 0,89 & 0,59 \\
\hline Maytenus aquifolia & 6 & 0,58 & 0,0221 & 0,05 & 10 & 0,89 & 0,51 \\
\hline Jacaranda puberula & 5 & 0,49 & 0,2121 & 0,49 & 6 & 0,54 & 0,50 \\
\hline Campomanesia guazumifolia & 5 & 0,49 & 0,0438 & 0,10 & 10 & 0,89 & 0,49 \\
\hline Aspidosperma australe & 5 & 0,49 & 0,0344 & 0,08 & 10 & 0,89 & 0,49 \\
\hline Annona sylvatica & 5 & 0,49 & 0,0938 & 0,22 & 8 & 0,72 & 0,47 \\
\hline Allophylus guaraniticus & 5 & 0,49 & 0,0574 & 0,13 & 8 & 0,72 & 0,44 \\
\hline Myrcia guianensis & 4 & 0,39 & 0,0424 & 0,10 & 8 & 0,72 & 0,40 \\
\hline
\end{tabular}


TABELA 2: Continuação...

TABLE 2: Continued...

\begin{tabular}{|c|c|c|c|c|c|c|c|}
\hline Táxons & DA & $\mathrm{DR}$ & DoA & DoR & FA & FR & VI \\
\hline Ilex dumosa & 3 & 0,29 & 0,0806 & 0,19 & 6 & 0,54 & 0,34 \\
\hline Styrax leprosus & 3 & 0,29 & 0,0518 & 0,12 & 6 & 0,54 & 0,32 \\
\hline Chomelia obtusa & 3 & 0,29 & 0,0091 & 0,02 & 6 & 0,54 & 0,28 \\
\hline Piptocarpha angustifolia & 3 & 0,29 & 0,0843 & 0,19 & 4 & 0,36 & 0,28 \\
\hline Ruprechtia laxiflora & 2 & 0,19 & 0,0947 & 0,22 & 4 & 0,36 & 0,26 \\
\hline Erythrina falcata & 1 & 0,10 & 0,1887 & 0,43 & 2 & 0,18 & 0,24 \\
\hline Cordia americana & 2 & 0,19 & 0,0671 & 0,15 & 4 & 0,36 & 0,24 \\
\hline Zanthoxylum rhoifolium & 2 & 0,19 & 0,0580 & 0,13 & 4 & 0,36 & 0,23 \\
\hline Eugenia uniflora & 2 & 0,19 & 0,0415 & 0,10 & 4 & 0,36 & 0,22 \\
\hline $\mathrm{NI}$ & 2 & 0,19 & 0,0305 & 0,07 & 4 & 0,36 & 0,21 \\
\hline Roupala montana & 2 & 0,19 & 0,0133 & 0,03 & 4 & 0,36 & 0,19 \\
\hline Myrocarpus frondosus & 2 & 0,19 & 0,0102 & 0,02 & 4 & 0,36 & 0,19 \\
\hline Terminalia australis & 2 & 0,19 & 0,0086 & 0,02 & 4 & 0,36 & 0,19 \\
\hline Myrsine sp. & 2 & 0,19 & 0,0073 & 0,02 & 4 & 0,36 & 0,19 \\
\hline Solanum pseudoquina & 2 & 0,19 & 0,0720 & 0,17 & 2 & 0,18 & 0,18 \\
\hline Cyatheaceae 1 & 3 & 0,29 & 0,0222 & 0,05 & 2 & 0,18 & 0,17 \\
\hline Lauraceae 1 & 1 & 0,10 & 0,1007 & 0,23 & 2 & 0,18 & 0,17 \\
\hline Myrcianthes gigantea & 1 & 0,10 & 0,0799 & 0,18 & 2 & 0,18 & 0,15 \\
\hline Myrcia bombycina & 2 & 0,19 & 0,0291 & 0,07 & 2 & 0,18 & 0,15 \\
\hline Citronella paniculata & 2 & 0,19 & 0,0264 & 0,06 & 2 & 0,18 & 0,14 \\
\hline Persea willdenowii & 1 & 0,10 & 0,0567 & 0,13 & 2 & 0,18 & 0,14 \\
\hline Eugenia ramboi & 2 & 0,19 & 0,0135 & 0,03 & 2 & 0,18 & 0,13 \\
\hline Gymnanthes concolor & 2 & 0,19 & 0,0113 & 0,03 & 2 & 0,18 & 0,13 \\
\hline Cordiera concolor & 1 & 0,10 & 0,0535 & 0,12 & 2 & 0,18 & 0,13 \\
\hline Clethra scabra & 1 & 0,10 & 0,0522 & 0,12 & 2 & 0,18 & 0,13 \\
\hline Albizia niopoides & 1 & 0,10 & 0,0509 & 0,12 & 2 & 0,18 & 0,13 \\
\hline Lauraceae 2 & 1 & 0,10 & 0,0504 & 0,12 & 2 & 0,18 & 0,13 \\
\hline Myrtaceae 1 & 1 & 0,10 & 0,0446 & 0,10 & 2 & 0,18 & 0,13 \\
\hline Sapium glandulosum & 1 & 0,10 & 0,0401 & 0,09 & 2 & 0,18 & 0,12 \\
\hline Trema micranta & 1 & 0,10 & 0,0268 & 0,06 & 2 & 0,18 & 0,11 \\
\hline Cinnamomum glaziovii & 1 & 0,10 & 0,0264 & 0,06 & 2 & 0,18 & 0,11 \\
\hline Vitex megapotamica & 1 & 0,10 & 0,0259 & 0,06 & 2 & 0,18 & 0,11 \\
\hline Vernonanthura discolor & 1 & 0,10 & 0,0229 & 0,05 & 2 & 0,18 & 0,11 \\
\hline Lamanonia ternata & 1 & 0,10 & 0,0193 & 0,04 & 2 & 0,18 & 0,11 \\
\hline Lithraea brasiliensis & 1 & 0,10 & 0,0161 & 0,04 & 2 & 0,18 & 0,10 \\
\hline Ocotea porosa & 1 & 0,10 & 0,0131 & 0,03 & 2 & 0,18 & 0,10 \\
\hline Xylosma tweediana & 1 & 0,10 & 0,0109 & 0,03 & 2 & 0,18 & 0,10 \\
\hline Myrsine guianensis & 1 & 0,10 & 0,0089 & 0,02 & 2 & 0,18 & 0,10 \\
\hline Drimys brasiliensis & 1 & 0,10 & 0,0088 & 0,02 & 2 & 0,18 & 0,10 \\
\hline Xylosma ciliatifolia & 1 & 0,10 & 0,0061 & 0,01 & 2 & 0,18 & 0,10 \\
\hline Banara parviflora & 1 & 0,10 & 0,0058 & 0,01 & 2 & 0,18 & 0,10 \\
\hline Zanthoxylum kleinii & 1 & 0,10 & 0,0042 & 0,01 & 2 & 0,18 & 0,10 \\
\hline Zanthoxylum fagara & 1 & 0,10 & 0,0029 & 0,01 & 2 & 0,18 & 0,09 \\
\hline Plinia trunciflora & 1 & 0,10 & 0,0026 & 0,01 & 2 & 0,18 & 0,09 \\
\hline Solanum pabstii & 1 & 0,10 & 0,0025 & 0,01 & 2 & 0,18 & 0,09 \\
\hline Cabralea canjerana & 1 & 0,10 & 0,0024 & 0,01 & 2 & 0,18 & 0,09 \\
\hline Sambucus australis & 1 & 0,10 & 0,0024 & 0,01 & 2 & 0,18 & 0,09 \\
\hline Total & 1027 & 100 & 43,57 & 100 & - & 100 & 100 \\
\hline
\end{tabular}

Em que: $\mathrm{DA}=$ densidade absoluta, em ind./ha; $\mathrm{DR}=$ densidade relativa, em \%; DoA = dominância absoluta, em $\mathrm{m}^{2} /$ ha; $\mathrm{DoR}=$ dominância relativa, em \%; FA = frequência absoluta, em \%; FR = frequência relativa, em \%.

inclusão da última parcela.

Tendo como referência outros estudos na região, a diversidade do remanescente estudado foi relativamente elevada $\left(\mathrm{H}^{\prime}=3,59\right)$ e a dominância baixa $\left(\mathrm{J}^{\prime}=0,80\right)$, com uma boa distribuição dos indivíduos por espécie, o que indica pequena dominância ecológica. Outros estudos na FOM têm demonstrado índice de diversidade de ShannonWiener em torno destes valores, como o de Higuchi et al. (2012a), que encontraram valores de $\mathrm{H}^{\prime}$ $=3,74$ e J' $=0,83$, e de Silva et al. (2012), com valores de $\mathrm{H}^{\prime}=3,6$ e $\mathrm{J}^{\prime}=0,82$. Nascimento et al. (2001), que encontraram a diversidade de 3,00, consideraram esse valor como sendo intermediário 


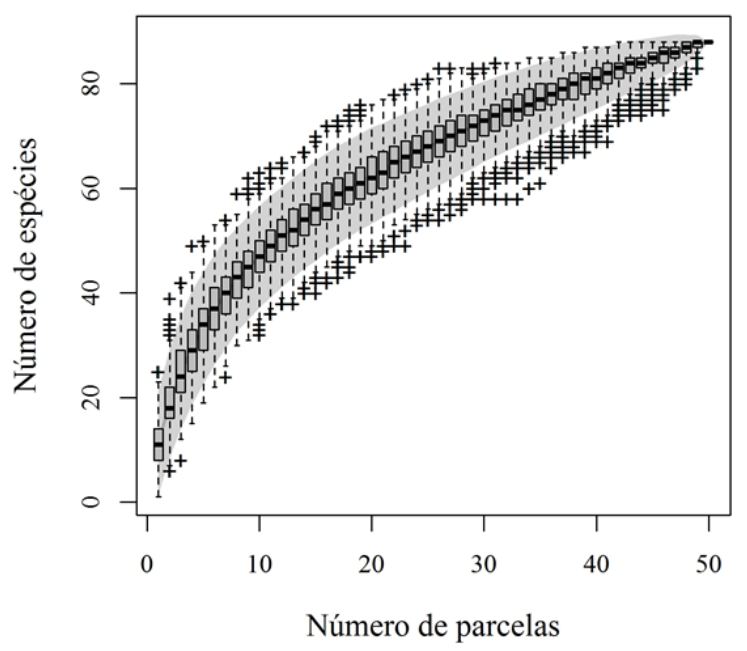

FIGURA 2: Curva de acumulação de espécies do componente arbóreo de um fragmento de Floresta Ombrófila Mista Montana em Campos Novos - SC.

FIGURE 2: Accumulation curve of the tree species component of a Montane Araucaria Forest in the municipality of Campos Novos - SC.

para o contexto da FOM com alguma intervenção antrópica. Silvestre et al. (2012), em um fragmento de FOM em Guarapuava, observaram um valor de $\mathrm{H}^{\prime}$ de 3,30, considerando o mesmo elevado. A baixa dominância ecológica indicada pelo índice de Pielou pode ser observada, também, nos baixos valores de densidade relativa (DR), sendo que somente Cupania vernalis possui DR maior que dez (11,68\%), resultando em uma alta equabilidade entre as espécies amostradas.

Os $1.027 \mathrm{ind} . /$ ha amostrados totalizaram uma área basal de $43,57 \mathrm{~m}^{2} / \mathrm{ha}$. A densidade pode ser considerada baixa e a área basal elevada se comparadas com dois outros remanescentes de FOM em Lages - SC, em que foram utilizados a mesma intensidade amostral e o mesmo nível de inclusão. No remanescente na localidade de Pedras Brancas (HIGUCHI et al., 2012a) foram encontrados 1.843 ind./ha, totalizando uma área basal de $36,45 \mathrm{~m}^{2} / \mathrm{ha}$, e na localidade Guará (SILVA et al., 2012) foram amostrados 1.783 ind./ha, resultando em uma área basal de $35,54 \mathrm{~m}^{2} / \mathrm{ha}$. No presente estudo, a elevada área basal, com uma menor densidade em relação à maior parte dos estudos de Lages, se deve a uma estruturação florestal caracterizada por árvores de grande porte, principalmente de araucária, que apresentou elevada dominância relativa $(35,50 \%)$, porém, com um número menor de indivíduos.

As três espécies com maior valor de importância (VI) foram Araucaria angustifolia (14,44\%), Cupania vernalis Cambess. $(8,82 \%)$ e Nectandra megapotamica (Spreng.) Mez (6,83\%), que são frequentemente relatadas entre as espécies com maiores valores de VI em fragmentos de FOM (e.g., SILVESTRE et al., 2012). Araucaria angustifolia, apesar do alto valor de VI, foi somente a sétima em maior densidade, com 40 indivíduos, e apresentou frequência absoluta de somente $44 \%$. No entanto, os 40 indivíduos de grande porte, com DAP médio de $67,19 \mathrm{~cm}$, somaram uma área basal de $15,4673 \mathrm{~m}^{2} /$ ha, que representa $35,5 \%$ da área basal de toda a comunidade. Assim, a elevada dominância desta espécie no remanescente estudado foi responsável pela elevada importância relativa da mesma na comunidade. Cupania vernalis apresentou o maior número de indivíduos (120) e a maior frequência nas parcelas estudadas (ocorreu em 82\% delas), porém, foi representada, principalmente, por indivíduos de porte menor, possuindo dominância relativa de 7,45\%. Nectandra megapotamica também apresentou mais do dobro do número de indivíduos que Araucaria angustifolia (90), mas também foi uma espécie representada predominantemente por árvores de menores diâmetros, com dominância relativa de $6 \%$.

Os valores médios das variáveis ambientais analisadas encontram-se na Tabela 3. De acordo, com a classificação proposta por Souza (2007), apesar de a declividade média indicar uma área com relevo ondulado $\left(9,53^{\circ}\right)$, existiu uma grande variação no remanescente, desde locais com a topografia plana $\left(0,57^{\circ}\right)$ até forte ondulada $\left(23,25^{\circ}\right)$. Considerando o contexto da área de ocorrência natural de Floresta Ombrófila Mista no Sul do Brasil (HIGUCHI et al., 2012b), a cota média pode ser considerada baixa, o que explica a influência de vários elementos típicos da Floresta Estacional. Em relação à fertilidade, representada pela saturação por bases $(\mathrm{V})$, o valor médio observado $(67,33 \% \pm 26,37 \%)$ foi superior ao encontrado em outros fragmentos da região, como, por exemplo, na localidade Pedras Brancas em Lages ( $\mathrm{V}=44,61 \%$ 19,03\%) (HIGUCHI et al., 2012a). Estes resultados sugerem que, regionalmente, os remanescentes de FOM ocorrem em um ambiente que apresenta elevada heterogeneidade edáfica, o que pode contribuir para explicar a substituição florística existente entre diferentes áreas (HIGUCHI et al., 2012b).

A análise NMDS obteve valor de stress 
TABELA3: Valores médios das variáveis ambientais analisadas em 50 parcelas alocadas em um remanescente de Floresta Ombrófila Mista Montana em Campos Novos - SC.

TABLE 3: Mean values of environmental variables analyzed within 50 plots allocated in a montane Araucaria Forest in Campos Novos - SC.

\begin{tabular}{lcc}
\hline \multicolumn{1}{c}{ Variáveis ambientais } & Média & $\begin{array}{c}\text { Desvio } \\
\text { Padrão }\end{array}$ \\
\hline Argila $(\%)$ & 26,72 & 5,18 \\
$\mathrm{pH}$ & 5,46 & 0,86 \\
$\mathrm{P}(\mathrm{mg} / \mathrm{dm})$ & 11,71 & 9,89 \\
$\mathrm{H}+\mathrm{Al}\left(\mathrm{cmolc} / \mathrm{dm}^{3}\right)$ & 6,85 & 8,08 \\
$\mathrm{Al}^{3+}\left(\mathrm{cmolc} \mathrm{dm}^{-3}\right)$ & 1,38 & 3,24 \\
$\mathrm{Ca}^{2+}\left(\mathrm{cmolc} / \mathrm{dm}^{3}\right)$ & 9,97 & 4,89 \\
$\mathrm{Na}^{3}\left(\mathrm{cmolc} / \mathrm{dm}^{3}\right)$ & 6,46 & 5,40 \\
$\mathrm{Mg}\left(\mathrm{cmolc} / \mathrm{dm}^{3}\right)$ & 2,12 & 0,96 \\
$\mathrm{~K}(\mathrm{mg} / \mathrm{dm})$ & 202,60 & 70,62 \\
$\mathrm{~V}(\%)$ & 67,33 & 26,37 \\
$\mathrm{SB}\left(\mathrm{cmolc} / \mathrm{dm}^{3}\right)$ & 12,64 & 5,76 \\
$\mathrm{CTC}$ efetiva $\left(\mathrm{cmolc} / \mathrm{dm}^{3}\right)$ & 20,87 & 8,38 \\
$\mathrm{CTC}$ a pH $=7\left(\mathrm{cmolc} / \mathrm{dm}^{3}\right)$ & 19,49 & 6,81 \\
Declividade média $\left(\mathrm{graus}^{2}\right)$ & 9,53 & 4,97 \\
Desnível máximo $(\mathrm{m})$ & 5,08 & 2,73 \\
Cota média $(\mathrm{m})$ & 729,32 & 31,62 \\
\hline
\end{tabular}

de 0,14 , indicando que o diagrama de ordenação construído é adequado para interpretação, de forma que a distância euclidiana das áreas nos diagramas está correlacionada de forma positiva com a dissimilaridade florística entre elas. Foi possível identificar um gradiente florísticoestrutural relacionado à cota média (altitude), $\mathrm{pH}$, saturação de bases e teor de $\mathrm{P}$ nos solos (Figura 3). O Eixo 1 representou as variações associadas, principalmente, à cota média, que indica o posicionamento das parcelas ao longo do gradiente topográfico, de áreas mais baixas até as mais elevadas. O Eixo 2 representou as variações associadas predominantemente pelo valores de $\mathrm{P}$ do solo. Destacaram-se nos locais de maior cota média, as espécies Solanum sanctaecatharinae Dunal e Matayba elaeagnoides Radlk. No outro extremo, nos locais de menor cota altimétrica, ocorrendo próximo do lago do reservatório da represa do Rio Canoas, Lonchocarpus campestris Mart. ex Benth. se destacou. As variáveis $\mathrm{pH}$ e saturação de base apresentaram correlação positiva e significativa entre si $(p<0,001)$ e definiram um gradiente com extremos de solos de maior fertilidade e básicos, no qual se destacaram Coutaria hexandra (Jacq.) K.Schum. e Araucaria angustifolia, e em solos mais ácidos e menos férteis, destacaram-se Dalbergia frutescens (Vell.) Britton, Luehea divaricata Mart. e Parapiptadenia rigida. Variações estruturais e florísticas do componente arbóreo associadas à cota

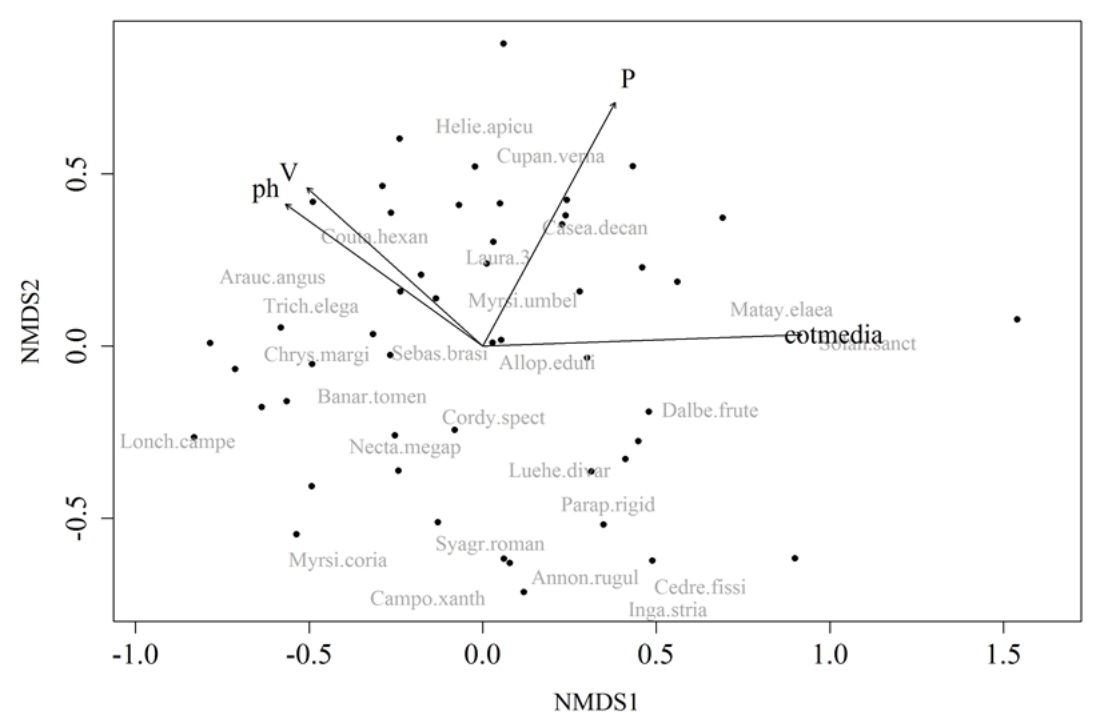

FIGURA 3: Distribuição das parcelas, das espécies e das variáveis ambientais estudadas em um remanescente de Floresta Ombrófila Mista Montana em Campos Novos - SC, na análise de NMDS (Nonmetric Multidimensional Scaling). cotmedia = cota média; $\mathrm{P}$ = teor de fósforo; $\mathrm{ph}=\mathrm{pH} ; \mathrm{V}=$ saturação de base.

FIGURE 3: Distribution of plots, of species and environmental variables studied in a remnant of Montane Araucaria Forest in Campos Novos - SC, in the analysis of NMDS (Nonmetric Multidimensional Scaling). cotmedia $=$ mean elevation; $\mathrm{P}=$ phosphorus content; $\mathrm{ph}=\mathrm{pH} ; \mathrm{V}=$ base saturation. 
e valores de $\mathrm{pH}$ do solo também foram observadas por Espírito-Santo et al. (2002) e Souza et al. (2003) em fragmentos de Floresta Estacional Semidecidual em Minas Gerais. Cupania vernalis, Helietta apiculata e Casearia decandra Jacq. ocorreram de forma preferencias nos locais com maiores valores de P no solo. Por outro lado, Lonchocarpus campestris, Campomanesia xanthocarpa O.Berg e Myrsine coriacea (Sw.) Roem. \& Schult. estiveram associadas, preferencialmente, a locais com baixos valores de P no solo.

\section{CONCLUSÕES}

No remanescente estudado foi encontrada alta diversidade de espécies e dominância ecológica relativamente baixa, destacando-se Araucaria angustifolia como a espécie de maior VI devido à maior dominância na área. Isso ocorreu porque esta espécie possui predominância de indivíduos de grande porte. A composição florística foi caracterizada pela mistura de elementos da Floresta Ombrófila Mista e da Floresta Estacional. Foram encontradas variações espaciais dos padrões florísticos e estruturais relacionados com a heterogeneidade ambiental existente. As espécies apresentaram variações em abundância relacionadas à cota média (altitude), $\mathrm{pH}$ e saturação de base dos solos, e teores de $\mathrm{P}$ nos solos. Considerando o contexto de recuperação de áreas degradadas, fica evidente que, para cada condição ambiental específica, existe um conjunto das espécies mais indicadas para serem utilizadas na recuperação.

\section{AGRADECIMENTOS}

Ao Conselho Nacional de Desenvolvimento Cientíco e Tecnológico-CNPq-Brasil, por financiar o projeto de pesquisa (Processo 479756/2007-4) e pelas bolsas de produtividade em pesquisa aos primeiro e segundo autores.

\section{REFERÊNCIAS BIBLIOGRÁFICAS}

ANGIOSPERM PHYLOGENY GROUP. An update of the Angiosperm Phylogeny Group classification for the orders and families of flowering plants: APG III. Botanical Journal of the Linnean Society, London, v. 161, n. 2, p. 105-121, 2009.

BABWETEERA, F.; BROWN, N. Can remnant frugivore species effectively disperse tree seeds in secondary tropical rain forests? Biodiversity and
Conservation, v. 18, n. 6, p. 1611-1627. 2009. BROWER, J. E.; ZAR, J. H. Field and laboratory methods for general ecology. Duduque: W. M. C. Brow Publishers, 1984. 84 p.

CLARK, D. B. Los fatores edáficos y la distribución de las plantas. In: GUARIGUATTA, M. R.; KATTAN, G. H. Ecología y conservación de bosques neotropicales. Cartago: Ediciones LUR, 2002, p. 192-221.

CORDEIRO, J.; RODERJAN, C.V.; CURCIO, G.R. Espécies lenhosas de um remanescente de Floresta Ombrófila Mista na região Centro-Sul e análise florística entre áreas florestais no Paraná. Ambiência, Guarapuava, v. 9, n. 3, p. 563-588, Set.-Dez., 2013.

EMBRAPA - EMPRESA BRASILEIRA DE PESQUISA AGROPECUÁRIA. Manual de métodos de análises de solo. Rio de Janeiro: Centro Nacional de Pesquisa de Solos, 1997. 212 p.

ESPÍRITO-SANTO, F. et al. Variáveis ambientais e a distribuição de espécies arbóreas em um remanescente de floresta estacional semidecídua montana no campus da Universidade Federal de Lavras, MG. Acta Botanica Brasilica, Feira de Santana, v. 16, n. 3, p. 331-356, jul.-set. 2002.

FERREIRA, P.I. et al. Espécies potenciais para recuperação de áreas de preservação permanente no Planalto Catarinense. Floresta e Ambiente, Seropédica, v. 20, n. 2, p. 173-182, abr.-jun, 2013.

HIGUCHI, P. et al. Influência de variáveis ambientais sobre o padrão estrutural e florístico do componente arbóreo, em um fragmento de Floresta Ombrófila Mista Montana em Lages, SC. Ciência Florestal, Santa Maria, v. 22, n. 1, p. 79-90, jan.-mar. 2012a.

HIGUCHI, P. et al. Floristic composition and phytogeography of the tree component of Araucaria Forest fragments in southern Brazil. Brazilian Journal of Botany, São Paulo, v. 35, n. 2, p. 145-157, abr.-jun. 2012b.

HIGUCHI, P. et al. Florística e estrutura do componente arbóreo e análise ambiental de um fragmento de Floresta Ombrófila Mista Altomontana no município de Painel, SC. Ciência Florestal, Santa Maria, v. 23, n.1 p. 153-164, jan.-mar. 2013.

HIJMANS, R. et al. Very high resolution interpolated climate surfaces for global land areas. International Journal of Climatology, v. 25, n. 15, p. 1965-1978. 2005.

IBGE. Manual técnico da vegetação brasileira. Série: Manual Técnico da Vegetação Brasileira. 
2 ed. Rio de Janeiro: IBGE, 2012. 271p.

JARENKOW, J.; WAECHTER, J. Composição, estrutura e relações florísticas do componente arbóreo de uma floresta estacional no Rio Grande do Sul, Brasil. Revista Brasileira de Botânica, São Paulo, v. 24, n. 3, p. 263-272, jul./set. 2001.

KLEIN, R. Mapa fitogeográfico de Santa Catarina. In: REITZ, P. Flora Ilustrada de Santa Catarina. Itajaí: Herbário Barbosa Rodrigues, 1978, p. 1-24.

KOZERA, C. et al. Fitossociologia do componente arbóreo de um fragmento de Floresta Ombrófila Mista Montana, Curitiba, PR, BR. Floresta, Curitiba, v. 36, n. 2, p. 225-237, 2006.

LINGNER, D. et al. Caracterização da estrutura e da dinâmica de um remanescente de Floresta com Araucária no Planalto Catarinense. Pesquisa Florestal Brasileira, Colombo, v. 55, p. 55-66, jul.-dez. 2007.

MINCHIN, P. R. An evaluation of relative robustness of techniques for ecological ordinations. Vegetatio, v. 71, p. 145-156. 1987.

MULLER-DOMBOIS, D.; ELLENBERG, H. Aims and of vegetation ecology. New York: John Wiley \& Sons, 1974. $547 \mathrm{p}$.

NASCIMENTO, A. R. T. et al. Estrutura e padrões de distribuição espacial de espécies arbóreas em uma amostra de Floresta Ombrófila Mista em Nova Prata, RS. Ciência Florestal, Santa Maria, v. 11, n. 1, p. 105-119, jan.-jun. 2001.

OKSANEN, J. et al. Vegan: community ecology package. R package version, v.1, p. 8-8. 2009.

OLIVEIRA FILHO, A. T. et al. Differenciation of streamside and upland vegetation in an area of montane semideciduous Forest in southeastern Brasil. Flora, v. 189, p. 1-19, 1994.

PUCHALSKI, Â. Variações edafo-climáticas e ocorrência natural de Araucaria angustifolia (Bert.) O. Kuntze no Estado de Santa Catarina. 2004. 85 f. Dissertação (Mestrado em Recursos Genéticos Vegetais), Universidade Federal de Santa
Catarina, Florianópolis.

R DEVELOPMENT CORE TEAM. R: A language and environment for statistical computing. R Foundation for Statistical Computing, 2008. Disponível em: <(http://www.R-project.org)> Acesso em: 18 de fevereiro de 2011.

SEGER, C. et al. Levantamento florístico e análise fitossociológica de um remanescente de floresta ombrófila mista localizado no município de Pinhais, Paraná-Brasil. Floresta, Curitiba, v. 35, n. 2, p. 291-302, mai.-ago. 2005.

SILVA, A. C. et al. Relações florísticas e fitossociologia de uma Floresta Ombrófila Mista Montana secundária em Lages, Santa Catarina. Ciência Florestal, Santa Maria, v. 22, n. 1, p. 193-206, jan.-mar. 2012.

SILVA, A. C. et al. Caracterização fitossociológica e fitogegráfica de um trecho de floresta ciliar em Alfredo Wagner, SC, como subsídio para restauração ecológica. Ciência Florestal, Santa Maria, v. 23, n. 4, p. 579-593, out.-dez., 2013.

SILVESTRE, R. et al. Análise estrutural e distribuição espacial em remanescente de Floresta Ombrófila Mista, Guarapuava (PR). Ambiência, Guarapuava, v. 8, n. 2, p. 259-274, mai.-ago., 2012.

SOUZA, C. G. Manual técnico de pedologia. Rio de Janeiro: Fundação Instituto Brasileiro de Geografia e Estatística (IBGE), Manuais Técnicos em Geociências, v. 4, 2007. 104 p.

SOUZA, J. et al. Análise das variações florísticas e estruturais da comunidade arbórea de um fragmento de Floresta Semidecídua às margens do rio Capivari, Lavras-MG. Revista Árvore, Viçosa, v. 27, n. 2, p. 185-206, mar.-abr. 2003.

VACCARO, S. et al. Aspectos da composição florística e categorias sucessionais do estrato arbóreo de três subseres de uma floresta estacional decidual, no município de Santa Tereza-RS. Ciência Florestal, Santa Maria, v. 9, n. 1, p. 1-18, jan.-jun. 1999. 